http://jmscr.igmpublication.org/home/ ISSN (e)-2347-176x ISSN (p) 2455-0450 crossref DOI: https://dx.doi.org/10.18535/jmscr/v7i8.01

\title{
A Comparative Study of the Effect of Fixed Versus Individualized Dialysate Sodium Levels on Blood Pressure and Interdialytic Weight Gain in Chronic Kidney Disease Patients on Maintenance Hemodialysis
}

\author{
Authors
}

\section{Dr Pulipati Akshaya Chowdary, Dr Sanju Rajappan*}

Department of Medicine, PES Institute of Medical Sciences \& Research Kuppam - 517425, A.P., India

*Corresponding Author

Dr Sanju Rajappan

Department of Nephrology, Malabar Medical College, Modakkallur, Calicut, Kerala 673323, India

\begin{abstract}
Hypertension is common in end-stage renal disease patients and accelerates cardiovascular morbidity and mortality. An important measure in achieving normal blood pressure in these patients is reaching the target dry weight. Sodium and extracellular fluid balance play a major role in blood pressure and interdialytic weight gain. Serum sodium levels have a role in both maintaining blood pressure and inter dialytic weight gain. Sodium concentration of the dialysate is critical in maintaining the serum sodium of the patient on dialysis and in ultra-filtrate removal. The standard method of single sodium level of dialysate for all patients ignores the inter-and intra-individual variability of serum sodium. The need for a study to assess the blood pressure changes using standard sodium in the dialysate and varying the sodium in the dialysate as per the pre hemodialysis serum sodium levels of the patient was anticipated. In this study we try to address such a concern. 30 patient on regular maintenance hemodialysis participated in the study, they underwent dialysis with standard levels of sodium (137meq/l) for 4 weeks, later for the next 4 weeks they underwent dialysis with a dialysate sodium level that was adjusted as per their pre hemodialysis serum sodium. The mean blood pressure during dialysis were $150.3 \mathrm{~mm} \mathrm{Hg}$ in the standard group and $148.3 \mathrm{~mm} \mathrm{Hg}$ in the individualized group. The mean intradialytic weight gain in the standardized group was $3.05 \mathrm{~kg}$ and in the individualized group was $3.02 \mathrm{~kg}$. The results did not show any significant difference in changes in blood pressure or inter dialytic weight gain.
\end{abstract}

\section{Introduction}

Hypertension is very common in patients with end-stage renal disease and accelerates cardiovascular morbidity and mortality. An important measure in achieving normotension is reaching the target dry weight. Sodium and extracellular fluid balance play a major role in blood pressure and interdialytic weight gain, and thus helps maintain dry weight. Sodium concentration of the dialysate is critical in providing such balance. The current use of single sodium level of dialysate for all patients ignores the inter- and intraindividual variability of serum sodium levels. This results in undesired consequences in $20-40 \%$ of cases $^{[1]}$, leading to episodes of hypotension during dialysis and unnecessary weigh gain inter dialysis.

If the dialysate sodium can be varied according to the patient's serum sodium levels the adverse 
effects can be reduced. To test this hypothesis the following study was done.

\section{Review of Literature}

Hypertension, a major consequence and cause of end-stage renal failure, remains persistent in a significant proportion of patients undergoing hemodialysis. The condition is seen in $90 \%$ of hemodialysis patients ${ }^{[1]}$, and its under-treatment may lead to a variety of cardiovascular diseases, e.g. left ventricular hypertrophy, cardiac chamber dilatation, inappropriate distribution of coronary blood flow, myocardial ischemia, myocardial fibrosis, heart failure, and arrhythmias. ${ }^{[2]}$ Reaching dry weight is considered as the first and most important factor in achieving normal blood pressure levels in these patients. Sodium and extracellular fluid balance play a major role in blood pressure and interdialytic weight gain. ${ }^{[3]}$

The intended function of dialysate fluid is to correct the composition of uremic blood to physiologic levels, both by reducing the concentration of uremic toxins and correcting electrolyte and acid-base abnormalities. This is accomplished by formulating a dialysate whose constituent concentrations are set to approximate normal values. Moreover, dialysate composition is a factor strongly affecting cardiovascular stability during treatment ${ }^{[4]}$. Composition of the dialysateis an essential element of dialysis prescription, in addition to dialyzer size, treatment time.blood and dialysate flow rates.

\section{Dialysate Sodium}

Sodium is the main extracellular ion and defines osmolality and size of the extracellular volume; increased plasma sodium concentration results in rise of osmolality, resulting in thirst and extracellular volume expansion. The latter results in arterial hypertension and left ventricular hypertrophy ${ }^{[5]}$.

Sodium homeostasis in hemodialysis (HD) patients is primarily dependent on two factors: dietary salt intake and sodium removal during dialysis. Salt intake during the interdialysis period is dependent on patient's behavior and awareness and is a strong driver of volume overload ${ }^{[6]}$.

NKF KDOQI guidelines recommend an upper limit of daily salt intake of $5 \mathrm{~g}(\sim 85 \mathrm{mmol}$ of sodium $)^{[7]}$. Despite the fact that dietary salt restriction is the most logical measure to prevent accumulation of salt and water in dialysis patients, it is not applied vigorously ${ }^{[8]}$. One of the primary goals of the dialysis therapy is to remove exactly the quantity of sodium that has accumulated in the interdialysis period in order to reach a zero sodium balance. This can be achieved through convection and diffusion process on dialysis. Currently HD rely primarily on convective and less on diffusive losses ${ }^{[9,10]}$. This relative distribution, however, is dependent on the amount of ultrafiltration occurring during any given dialysis session (i.e. convective losses), and the prescribed dialysate sodium concentration (dial $\mathrm{Na}+$ ) and its relationship with the patient's own plasma sodium (the inlet dialyzer diffusion concentration gradient between dialysate and

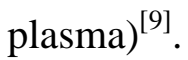

In the 1980s, hydrostatic ultrafiltration was applied, where dial $\mathrm{Na}+$ was $\sim 136 \mathrm{mmol} / \mathrm{L}$ and the dialysis time $4-5$ hours. During the last few years, there remains widespread acceptance of higher dial $\mathrm{Na}+(>140 \mathrm{mmol} / \mathrm{L})$ and sodium modelling in order to avoid hemodynamic instability during the shortened dialysis treatment [11].

A number of options of dial $\mathrm{Na}+$ are currently being used in daily practice including fixed, low or high dial $\mathrm{Na}+$ or variable (individualized) dial $\mathrm{Na}+$ (e.g. dial $\mathrm{Na}+$ tailored to serum concentrations, sodium modelling strategies or online monitoring of plasma conductivity with automatic adjustment of dialysate conductivity) ${ }^{[12]}$.

\section{Sodium - interdialytic weight gain and hypertension}

A recent report from the Dialysis Outcomes and Practice Patterns Study (DOPPS) showed that the majority of HD facilities (57\%) adopted uniform rather than variable dial $\mathrm{Na}+$ prescriptions in more 
than $90 \%$ of patients ${ }^{[13]}$. Nevertheless, the issue as to whether low or high fixed dial $\mathrm{Na}+$ prescriptions should be advocated in chronic HD patients is still debated. High dial $\mathrm{Na}+$ prescriptions can be useful for preventing hypotensive episodes but lead to a positive sodium mass balance that increase blood pressure and weight gain by increased thirst. Conversely, low dial $\mathrm{Na}+$ prescription reduce thirst, blood pressure and weight gain but can be harmful, particularly in hypotension-prone subjects ${ }^{[14]}$. It has been postulated that each individual has an inherent plasma sodium concentration or 'sodium set point ${ }^{[15]}$. Hence, increase in the serum sodium concentration as a result of dietary intake and hemodialysis treatment is followed by a thirstdriven water intake to reduce the osmolality until the corresponding serum sodium concentration is reached. A strong association has been demonstrated between thirst and intradialytic weight gain $^{[16]}$. Increased thirst and water intake in patients require higher ultrafiltration rates in order to remove this excess fluid during the next hemodialysis session.

Not only high dial $\mathrm{Na}+$ is hypertensionogenic, but also the converse is true: low dial $\mathrm{Na}+$ leads to better sodium balance and BP control. Historical data indicate much better BP control when dialysate sodium concentration was much lower than presently. However, several other differences were operative, most importantly duration of dialysis, which is an independent determinant of BP control regardless of degree of volume control $^{[17]}$.

\section{Intradialytic Hypotension}

A sudden and symptomatic fall in BP during a dialysis session is called Intradialytic hypotension. During ultrafiltration, fluid is removed from the vascular space. This requires prompt refilling from the extracellular fluid to maintain blood volume. Healthy adults will tolerate upto $25 \%$ reduction in blood volume, but, patients on HD, there is large inter- and intra- patient variability.
Intradialytic hypotension is defined as a fall in systolic $\mathrm{BP}(\mathrm{SBP})>20 \mathrm{~mm}$ of $\mathrm{Hg}$ or mean arterial pressure $>10 \mathrm{~mm} \mathrm{Hg}$, associated with symptoms, or a fall to SBP $<100 \mathrm{~mm} \mathrm{Hg}$.

Symptoms associated with intradialytic hypotension include cramps, abdominal pain, nausea (reduced gut perfusion) ,yawning, sighing, anxiety ,dizziness (reduced cerebral perfusion) and chest pain , arrhythmias.

\section{Aims and Objectives}

1) To perform dialysis with standardized dialysate sodium of $140 \mathrm{mmol} / \mathrm{L}$. for all patients and with dialysate sodium individualized to the serum sodium of each patient for 4 weeks each.

2) To document blood pressure (BP) changes during dialysis

3) To document weight gain and control of hypertension in interdialytic periods

\section{Materials and Methods}

Study design: Hospital based prospective study Sample size: 30 patients

Method: All Patients will be subjected to dialysis with fixed dialysate sodium of $140 \mathrm{mmol} / \mathrm{L}$ for a period of 4 weeks and individualized dialysate sodium, sodium levels in the dialysate chosen as per the serum levels of the patients for 4 weeks. One serum sodium level shall be done at the beginning of dialysis. All patients to undergone dialysis with fixed sodium dialysate and individualized dialysate sodium.

Monitoring of blood pressure during dialysis will be done every half hourly and the mean BP calculated for each session of dialysis and the mean of the 4 weeks shall be taken for computation

Inter dialytic weight gain to be documented before each dialysis and the mean of the 4 weeks to be used for computation

Measurement of blood pressure at the start of dialysis is taken as the blood pressure during the inter dialytic period and shall be used in computation after calculating the mean 
Conductivity on the dialysis was used for achieving required dialysate sodium.

Study period: 18 months (January 2015 to October 2017).

Equipment: NIKKISO DBB27 Hemodialyser, spghymomanometer

\section{Inclusion Criteria}

- Adult chronic kidney disease patients on maintenance hemodialysis (HD) for at least 3 months.

\section{Exclusion Criteria}

- Acute kidney injury

- Acute on chronic kidney disease

- Serum Sodium levels $<135$ and $>150$ $\mathrm{mmol} / \mathrm{L}$

- History of Arrhythmias

Analysis of data: Data were entered in MS Excel 2007 and analyzed by STATA v14 software. Inferential statistics was done using t-test for means \pm standard deviations, where $\mathrm{p}$ - value $<0.05$ was statistically significant

\section{Observations and Results}

Total of 30 patients (21 males and 9 females) completed the study.

Table 1: Mean serum pre HD sodium

\begin{tabular}{|l|c|}
\hline Subject & Serum pre HD Na+ mmol/L \\
\hline 1 & 137.9444 \\
\hline 2 & 142.6111 \\
\hline 3 & 139.0588 \\
\hline 4 & 138.4444 \\
\hline 5 & 142.8889 \\
\hline 6 & 141.6667 \\
\hline 7 & 138.1176 \\
\hline 8 & 135.7647 \\
\hline 9 & 139.7368 \\
\hline 10 & 138.0556 \\
\hline 11 & 143.4444 \\
\hline 12 & 146.1667 \\
\hline 13 & 143.5 \\
\hline 14 & 140.9444 \\
\hline 15 & 141.6111 \\
\hline 16 & 142.05 \\
\hline 17 & 143.9412 \\
\hline 18 & 140.8889 \\
\hline 19 & 142.2632 \\
\hline 20 & 136.9375 \\
\hline 21 & 142.125 \\
\hline 22 & 138.2632 \\
\hline 23 & 138.9333 \\
\hline 24 & 143.5882 \\
\hline 25 & 140.1875 \\
\hline & \\
\hline
\end{tabular}

\begin{tabular}{|l|c|}
\hline 26 & 144.5 \\
\hline 27 & 143.875 \\
\hline 28 & 136.8125 \\
\hline 29 & 141.75 \\
\hline 30 & 138.1875 \\
\hline Mean & $\mathbf{1 4 0 . 8 0 8 6}$ \\
\hline
\end{tabular}

Table 2: Mean of Blood pressure

\begin{tabular}{|c|c|c|c|c|}
\hline \multirow{2}{*}{$\begin{array}{l}\text { Subjec } \\
\text { t }\end{array}$} & \multicolumn{2}{|c|}{ SBP $(\mathbf{m m}$ of $\mathbf{H g})$} & \multicolumn{2}{|c|}{ (DBP $\mathbf{~ m m}$ of $\mathbf{H g}$ ) } \\
\hline & $\begin{array}{c}\text { Standar } \\
\text { d HD }\end{array}$ & $\begin{array}{c}\text { Individualize } \\
\text { d Na+ HD }\end{array}$ & $\begin{array}{c}\text { Standar } \\
\text { d HD }\end{array}$ & $\begin{array}{c}\text { Individualize } \\
\text { d Na+ HD }\end{array}$ \\
\hline 1 & 145 & 147.5 & 88 & 87.5 \\
\hline 2 & 144.4 & 150.2 & 88 & 86.66 \\
\hline 3 & 147.75 & 139.5 & 83.5 & 83.25 \\
\hline 4 & 137.11 & 129.11 & 77.33 & 74.44 \\
\hline 5 & 142.5 & 145 & 79.75 & 88.5 \\
\hline 6 & 150.5 & 145 & 82.6 & 86.5 \\
\hline 7 & 148.25 & 143.75 & 88.25 & 82.75 \\
\hline 8 & 167.25 & 148.25 & 88.75 & 81.75 \\
\hline 9 & 158.22 & 146 & 88.88 & 87.55 \\
\hline 10 & 139.75 & 158.5 & 86 & 86 \\
\hline 11 & 141.25 & 140.25 & 82.25 & 79.25 \\
\hline 12 & 142.5 & 162.25 & 89.25 & 90.75 \\
\hline 13 & 145.11 & 159.77 & 83.11 & 88.66 \\
\hline 14 & 160.5 & 149.75 & 83.75 & 87 \\
\hline 15 & 159.75 & 157.5 & 82.25 & 87 \\
\hline 16 & 155.6 & 162.2 & 89.4 & 88.6 \\
\hline 17 & 149.75 & 162.5 & 84 & 84.25 \\
\hline 18 & 158 & 133.75 & 86.5 & 89 \\
\hline 19 & 147.77 & 150.66 & 87.11 & 86.44 \\
\hline 20 & 151.42 & 146.85 & 92.57 & 81.42 \\
\hline 21 & 149.42 & 155.42 & 83.71 & 88.28 \\
\hline 22 & 137.11 & 150.88 & 80.88 & 88.44 \\
\hline 23 & 152.85 & 149.14 & 90 & 80.28 \\
\hline 24 & 155.5 & 145 & 101.75 & 87 \\
\hline 25 & 146.25 & 150.25 & 87.75 & 87.5 \\
\hline 26 & 150.25 & 139.5 & 81.75 & 83.25 \\
\hline 27 & 167.25 & 148.25 & 88.75 & 81.75 \\
\hline 28 & 148.25 & 145.25 & 88.25 & 85.25 \\
\hline 29 & 156.5 & 152.25 & 90 & 86.25 \\
\hline 30 & 150.5 & 146.5 & 82.5 & 83.75 \\
\hline
\end{tabular}

Table 3: Mean IDWG

\begin{tabular}{|l|c|c|}
\hline \multirow{2}{*}{ Subject } & \multicolumn{2}{|c|}{ IDWG (Kg) } \\
\cline { 2 - 3 } & Standard HD & Individualized Na+ HD \\
\hline 1 & 2.64 & 2.00 \\
\hline 2 & 3.46 & 3.68 \\
\hline 3 & 3.57 & 2.77 \\
\hline 4 & 1.32 & 2.30 \\
\hline 5 & 3.42 & 3.77 \\
\hline 6 & 1.84 & 1.85 \\
\hline 7 & 3.02 & 2.88 \\
\hline 8 & 3.04 & 2.85 \\
\hline 9 & 4.15 & 3.01 \\
\hline 10 & 3.02 & 2.61 \\
\hline 11 & 1.95 & 2.07 \\
\hline 12 & 4.10 & 4.02 \\
\hline 13 & 4.00 & 4.05 \\
\hline 14 & 3.85 & 4.14 \\
\hline 15 & 2.41 & 2.71 \\
\hline 16 & 2.86 & 3.41 \\
\hline 17 & 2.91 & 2.98 \\
\hline 18 & 1.06 & 1.02 \\
\hline 18 & 3.22 & 3.65 \\
\hline
\end{tabular}




\begin{tabular}{|l|l|l|}
\hline 20 & 3.38 & 3.48 \\
\hline 21 & 3.53 & 3.18 \\
\hline 22 & 2.64 & 3.22 \\
\hline 23 & 3.28 & 3.40 \\
\hline 24 & 3.39 & 3.28 \\
\hline 25 & 3.67 & 3.85 \\
\hline 26 & 3.18 & 2.95 \\
\hline 27 & 1.98 & 1.85 \\
\hline 28 & 4.50 & 4.52 \\
\hline 29 & 2.18 & 2.10 \\
\hline 30 & 4.00 & 2.50 \\
\hline
\end{tabular}

Statistical analysis for continuous data which followed normal distribution is performed using paired student $t$ test

Table 4: SBP - Statistical data

\begin{tabular}{|l|c|c|}
\hline SBP & $\begin{array}{c}\text { MEAN } \\
\text { (mm of Hg) }\end{array}$ & $\begin{array}{c}\text { STANDARD } \\
\text { DEVIATION }\end{array}$ \\
\hline Standard HD & 150.3055 & 7.755312 \\
\hline Individualized Na+ HD & 148.3465 & 7.896319 \\
\hline
\end{tabular}

The $P$ value is 0.3332

Table 5: DBP - Statistical data

\begin{tabular}{|l|c|c|}
\hline DBP & $\begin{array}{c}\text { MEAN } \\
\text { (mm of Hg) }\end{array}$ & $\begin{array}{c}\text { STANDARD } \\
\text { DEVIATION }\end{array}$ \\
\hline Standard HD & 86.04159 & 4.587542 \\
\hline $\begin{array}{l}\text { Individualized Na+ } \\
\text { HD }\end{array}$ & 85.24262 & 3.557187 \\
\hline
\end{tabular}

The $\mathrm{P}$ value is 0.4135

The decrease in SBP and DBP in both the groups is not statistically significant

Table 6: IDWG - Statistical data

\begin{tabular}{|l|c|c|}
\hline IDWG & $\begin{array}{c}\text { MEAN } \\
(\mathbf{K g})\end{array}$ & $\begin{array}{c}\text { STANDARD } \\
\text { DEVIATION }\end{array}$ \\
\hline Standard HD & 3.055089 & 0.8494956 \\
\hline Individualized Na+ HD & 3.022073 & 0.7926176 \\
\hline
\end{tabular}

The $P$ value is 0.7111

The difference in IDWG in both the groups is very minimal and is statistically insignificant.

There were no intradialytic hypotensive episodes in both groups.

\section{Discussion}

Chronic kidney disease (CKD) is often an asymptomatic condition resulting in dependence on renal replacement therapy at a later stage. Although, renal transplantation is an excellent option, there are shortcomings including lack of donors and cost. Dialysis is then adopted for survival in these individuals. CKD is one of the most important causes for secondary and resistant hypertension. Control of hypertension is essential in reducing mortality, though it is difficult to maintain $\mathrm{Na}+$ homeostasis, which not only affects the BP, but also causes fluid retention. Fluid retention causes increased preload and pulmonary congestion, affecting the quality of life. Hence, it is very important that the HD performed is adequate and near physiological.

The concept of an individual and relatively 'fixed' osmolar set point in HD patients is crucial to understand sodium balance. Humans have mechanisms to preserve their extracellular osmolality towards a seemingly fixed set point, and it appears that HD patients also maintain a fixed osmolar set point despite the loss of kidney and vasopressin feedback mechanism. ${ }^{[17]}$ Furthermore, pre-HD plasma sodium concentrations are quite reproducible, suggesting that there is a preferred plasma sodium concentration in individual HD patients ; furthermore, plasma sodium concentration in HD patients is stable in long-term observations. ${ }^{[18]}$

Until the introduction of controlled ultrafiltration in the 1970s, dextrose-containing hyponatremic dialysis fluids were prescribed to facilitate water and sodium removal by osmotic and diffusive gradients. ${ }^{[17]}$ Although nowadays most of the excess sodium is removed by ultrafiltration, diffusive sodium removal still has an important clinical impact on the total intradialytic sodium balance. ${ }^{[18]}$ High dialysate sodium levels have been advocated to reduce intradialytic hypotensive episodes and symptoms such as cramping and nausea ${ }^{[36]}$, but are associated with fluid overload and hypertension ${ }^{[8,37]}$, and to left ventricular hypertrophy and cardiovascular events. Low dialysate concentrations have improved volume and blood pressure control, but may lead to more intradialytic symptoms. The optimal dialysate sodium concentration is different for each individual and largely depends on the sodium concentration gradient, i.e. the difference between dialysate sodium and pre- HD sodium. 
This study of 30 patients at a rural tertiary care center on the effect of individualized sodium prescription to the standard sodium prescription in $\mathrm{HD}$, with respect to BP, IDWG and intradialytic symptoms. The advantage of conducting a shortterm study is that all the important parameters like estimated dry weight and the dosage of drugs, remain the same and thereby the observed changes are exclusively due to the change in dial $\mathrm{Na}+$

In terms of the number of patients studied, current study is on par with similar studies .The average pre HD Na+ in our study is $140.8086 \mathrm{mmol} / \mathrm{L}$ and we have used $140 \mathrm{mmol} / \mathrm{L}$ as the standard dial $\mathrm{Na}+$. When individualizing the Dial $\mathrm{Na}+$ we have used the pre HD $\mathrm{Na}+$ as the reference for Dial $\mathrm{Na}+$, which looked more physiological.

\section{Blood Pressure}

Blood pressure control during the interdialytic period is considered as the $\mathrm{BP}$ of the person before the dialysis. The mean BP with standard dial $\mathrm{Na}+$ was $150.30 / 86.04 \mathrm{~mm}$ of $\mathrm{Hg}$ and after individualized dial $\mathrm{Na}+$ was $148.35 / 85.24 \mathrm{~mm}$ of $\mathrm{Hg}$. There was a reduction in the mean BP of the individuals as in agreement with other studies, however, it failed to achieve statistical significance.

The results of the current study are concordant with those of Selby et al and Koonan et al. However, the patients were not adherent to dietary recommendations and $\mathrm{Na}+$ restriction in the diet and needs education for the same. Probably a larger and longer study is required to achieve statistical significance.

\section{Interdialytic Weight Gain}

Interdialytic weight gain is the weight gained during the interdialytic period and is dependent on intake of salt and water. Higher salt intake stimulates thirst and hence, water intake. In our study, the IDWG in the group with standard dial $\mathrm{Na}+$ is $3.055 \mathrm{Kg}$ and in the group with individualized dial $\mathrm{Na}+$ is $3.022 \mathrm{Kg}$, with no statistical difference. There is an agreement of the above results with the studies by Farmer et al, Kooman et al and Selby et al., but not so with other studies performed by De Paula et al, Lambie et al, Sayarlioglu et al, Thein et al and Yasser Elshahawy et $\mathrm{al}^{[24]}$. The variation may be attributed to the climate and the occupation of the study population who perform daily labour and are prone to excessive losses in hot environments and lack of strict adherence to dietary recommendations.

\section{Intradialytic Hypotension}

In the current study there were no intradialytic events in both the groups, perhaps the period of observation was not long enough. However, some studies demonstrated the reduction of intradialytic symptoms in the interventional group.

Our study is a short term single center prospective study, on hypertensive dialysis patients, using simple, technique for control of dial $\mathrm{Na}+$ that is easily reproducible with every patient being his control, the bias is minimal. There was no proven or potential harm because of the intervention performed, however poor awareness regarding diet and the disease, contributed to increased intake of salt and water.

Further studies need to be performed for a longer duration to look for long term effects and with larger number of patients so as to identify specific sub groups of patients who may benefit with individualization of dial $\mathrm{Na}+$.

\section{Conclusion}

There is a beneficial reduction in blood pressure, but not of statistical significance. There has been no change in IDWG and intradialytic hypotension in the two groups. Individualization of dialysate appeared to be safe, however, long term benefits need to be ascertained. There is a need to reconsider the dial $\mathrm{Na}+$ levels so as to provide better dialytic care and need for screening patients most likely to benefit from this approach .

Sources of support in the form of grants: None.

\section{References}

1. Chang TI. Systolic blood pressure and mortality in patients on hemodialysis. Curr Hypertens Rep. 2011; 13:362-9. 
2. Brenner BM, Maarten W, Taal GMC, Philip A, Marsden Karl Skorecki, Alan SL YU. Lippincott, Williams and Wilkins: Elsevier; 2012. The Kidney; p. 2294. 2296,2322,2324,2331.

3. Frankliu, Jon H, LaraghCharra B. Core Curriculum Fluid Balance, dry weight, and blood pressure in dialysis. Hemodial Int. 2007;11:21-31.

4. Locatelli F, Covic A, Chazot C, et al. Optimal composition of the dialysate, with emphasis on its influence on blood pressure. Nephrol Dial Transplant 2004; 19: 785-796

5. Cook NR, Cutler JA, Obarzanek E, et al. Long term effects of dietary sodium reduction on cardiovascular disease outcomes: observational follow-up of the Trials of Hypertension Prevention (TOHP). BMJ 2007; 334: 885-888

6. Santos SFF, Peixoto AJ. Sodium balance in maintenance hemodialysis. Semin Dial 2010; 23: 549-555

7. NKF KDOQI Guidelines. Hemodialysis adequacy, update 2006. Am J Kidney Dis 2006; 48 (Suppl 1): S2-S90

8. Oke. How to successfully achieve salt restriction in dialysis patients? What are the outcomes? Blood Purif 2010; 29: 102104

9. Basile C, Libutti P, Lisi P, et al. Sodium setpoint and gradient in bicarbonate hemodialysis. J Nephrol2013; 26: 11361142

10. Odudu A, Lambie SH, Taal MW, et al. Use of online conductivity monitoring to study sodium mass balance in chronic hemodialysis patients: prospects for treatment individualisation. Kidney Blood Press Res 2011; 34: 439-446

11. Sam R, Vaseemuddin M, Leong WH, et al. Composition and clinical use of hemodialysates. HemodialInt 2006; 10: $15-28$
12. McCausland FR, Tilley BS, Waikar SS. Dialysate sodium and the milieu intérieur. Clin J Am SocNephrol 2012; 7: 5-7

13. Yasser Elshahawy, DawlatSany, Sahar Shawky; Outcome of Individualized Dialysate Sodium Concentration for Hemodialysis Patients: Saudi J Kidney Dis Transpl 2013;24(3):507-513, 2013 Saudi Center for Organ Transplantation

14. Levin NW, Zhu F, Keen M: Interdialytic weight gain and dry weight. Blood Purif 2001; 19: 217-221.

15. Bots CP, Brand HS, Veerman EC, et al: Interdialytic weight gain in patients on hemodialysis is associated with dry mouth and thirst. Kidney Int 2004; 66: 1662 1668.

16. Sergio F. F. Santos, and Aldo J. Peixoto; Revisiting the Dialysate Sodium Prescription as a Tool for Better Blood Pressure and Interdialytic Weight Gain Management in Hemodialysis Patients; Clinical Journal of the American Society Nephrology 3: 522-530, 2008. doi: 10.2215/CJN.03360807.

17. Bourque CW. Central mechanisms of osmosensation and systemic osmoregulation. Nat Rev Neurosci 2008; 9: 519-531

18. Peixoto AJ, Gowda N, Parikh CR et al. Long-term stability of serum sodium in hemodialysis patients. Blood Purif 2010; 29: 264-267

19. Mann H, Stiller S: Sodium modeling. Kidney IntSuppl 2000; 76:S79-S88

20. Henrich WL, Woodard TD, McPhaul JJ Jr: The chronic efficacy and safety of high sodium dialysate: double-blind, crossover study. Am J Kidney Dis 1982; 2: 349-353.

21. Gotch FA, Lam MA, Prowitt M, Keen M: Preliminary clinical results with sodium volume modeling of hemodialysis therapy. ProcClin Dial Transplant Forum 1980; 10: 12-17. 
22. de Paula FM, Peixoto AJ, Pinto LV, Dorigo D, Patricio PJ, Santos SF: Clinical consequences of an individualized dialysate sodium prescription in hemodialysis patients. Kidney Int 66: 1232-1238, 2004

23. Sayarlioglu H, Erkoc R, Tuncer M, Soyoral Y, EsenR,Gumrukcuoglu HA, Dogan E, Sayarlioglu M: Effects of low sodium dialysate in chronic hemodialysis patients: an echocardiographic study. Ren Fail 29: 143-146, 2007

24. Thein H, Haloob I, Marshall MR: Associations of a facility level decrease in dialysate sodium concentration with blood pressure and interdialytic weight gain. Nephrol Dial Transplant 22: 2630-2639, 2007

25. NKF-KDOQI, update 2015, sec III.

\section{List of Abbreviations}

(In order of appearance)

BP - Blood pressure

HD - Hemodialysis

mmol - Milli-moles

NKF KDOQI - National Kidney Foundation

Kidney Disease Outcomes Quality

Initiative

Dial Na+ - Dialysate sodium

L - Liter

DOPPS - Dialysis Outcomes and Practice Patterns

Study

USA - United States of America

IDWG - Interdialytic weight gain

$\mathrm{mm}$ of $\mathrm{Hg}$ - Milli-meters of Mercury

SBP - Systolic blood pressure

DBP - Diastolic blood pressure

$\mathrm{Na}+$ - Sodium

CKD - Chronic Kidney Disease

No. - Number

Fig - Figure

$\mathrm{SD}$ - Significant decrease

NS - Not significant

$\mathrm{Kg}-$ Kilogram 